\title{
Crystal structure of 2',4'-dimethoxy-3-hydroxyflavone, $\mathrm{C}_{17} \mathrm{H}_{14} \mathrm{O}_{5}$
}

\author{
M. Shoja \\ Fordham University. Department of Chemistry, Fordham Road, Bronx, NY 10458, USA
}

Received December 11, 2000, CCDC-No. $1267 / 583$

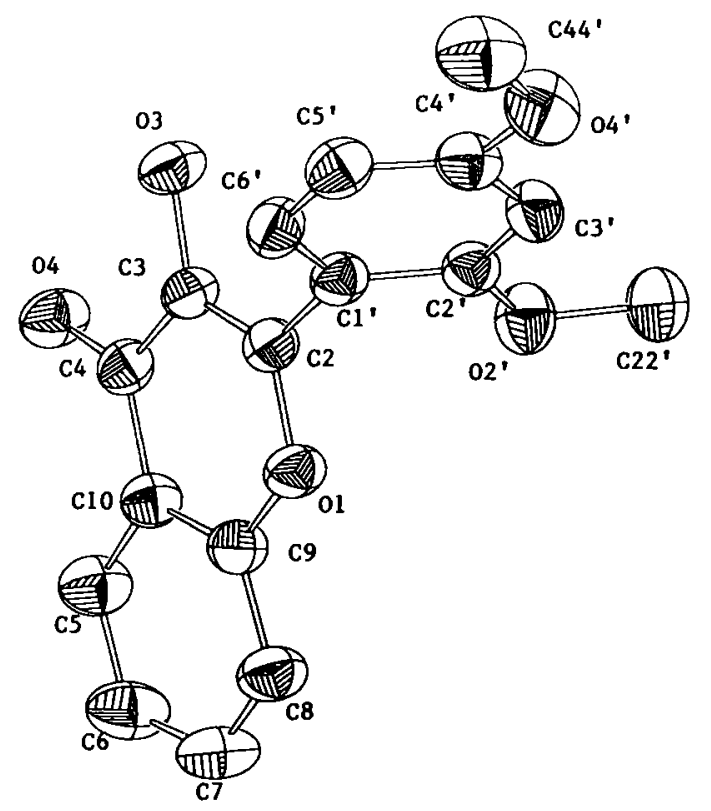

\section{Abstract}

$\mathrm{C}_{17} \mathrm{H}_{14} \mathrm{O}_{5}$, triclinic, $P \overline{\mathrm{I}}$ (No. 2), $a=7.377(3) \AA, b=9.619(3) \AA$, $c=11.901(3) \AA, \alpha=107.70(1)^{\circ}, \beta=100.06(1)^{\circ}, \gamma=108.77(1)^{\circ}$, $V=726.2 \AA^{3}, Z=2, R_{\mathrm{gt}}(F)=0.037, w R_{\mathrm{all}}(F)=0.043, T=293 \mathrm{~K}$.

\section{Source of material}

Crystals of the title compound (Indofine Chemical Company) were grown from ethyl acetate solution.

\section{Discussion}

The X-ray crystal structure of the title compound was determined to show the effect of the repulsion of the lone-pair electrons of the methoxy $\mathrm{O}_{2}$ ' and the hydroxy $\mathrm{O} 3$ atoms, which will cause a twist between the phenyl ring and the $\gamma$-pyrone portion of the molecule. The torsion angle for $\mathrm{C} 3-\mathrm{C} 2-\mathrm{Cl}^{\prime}-\mathrm{C}^{\prime}$ is $-113.2(3)^{\circ}$. This repulsion was also observed in 3-hydroxy-2'-methoxyflavone with a torsion angle of $72.2(4)^{\circ}[1]$. In the title compound, the $\mathrm{C} 2-\mathrm{Cl}^{\prime}$ bond distance is 1.473(4) $\AA$. A weak bifurcated hydrogen bond is formed as a result of an inter- $(d(\mathrm{O} 4 \cdots \mathrm{H} 3)=2.31 \AA)$ and an intra-molecular $(d(\mathrm{O} 4 \cdots \mathrm{H} 3)=2.05 \AA)$ attraction between the molecules. We have reported the same type of hydrogen bond for 3-hydroxy-6-methoxy and 3-hydroxy-7-methoxyflavone with torsion angles of $11.3(5)^{\circ}$ and $1.2(6)^{\circ}$, respectively $[2,3]$. In conclusion, the methoxy group in the $\mathrm{C}^{\prime}$ ' position in the presence of a C3 hydroxy group will cause a twist around the $\mathrm{C} 2-\mathrm{Cl}^{\prime}$ bond, and pack the molecule in a non-planar conformation.

Table 1. Data collection and handling.

Crystal:

Wavelength:

$\mu$ :

Diffractometer, scan mode:

$2 \theta_{\max }$ :

$N(h k l)_{\text {measured, }} N(h k l)_{\text {unique: }}$

Criterion for $F_{\text {obs, }} N(h k l)_{g t}$ :

$N(\text { param })_{\text {refined: }}$

Program: pale yellow needle, size $0.3 \times 0.3 \times 0.4 \mathrm{~mm}$

Mo $K_{\alpha}$ radiation $(0.71073 \AA$ )

$1.1 \mathrm{~cm}^{-1}$

CAD4, $\omega / 2 \theta$

$49.94^{\circ}$

2736,2545

$F_{\text {obs }}>3 \sigma\left(F_{\text {obs }}\right), 1989$

255

XTAL [4]

Table 2. Atomic coordinates and displacement parameters (in $\AA^{2}$ ).

\begin{tabular}{llllll}
\hline Atom & Site & $x$ & $y$ & $z$ & $U_{\text {iso }}$ \\
\hline$H\left(6^{\prime}\right)$ & $2 i$ & $0.355(3)$ & $0.987(3)$ & $0.122(2)$ & $0.044(7)$ \\
$\mathbf{H}\left(3^{\prime}\right)$ & $2 i$ & $0.608(4)$ & $1.246(3)$ & $0.550(3)$ & $0.059(8)$ \\
$\mathbf{H}(8)$ & $2 i$ & $0.547(4)$ & $0.529(3)$ & $0.285(2)$ & $0.063(8)$ \\
$\mathbf{H}\left(5^{\prime}\right)$ & $2 i$ & $0.220(4)$ & $1.160(3)$ & $0.225(2)$ & $0.053(7)$ \\
$\mathbf{H}(5)$ & $2 i$ & $0.862(4)$ & $0.538(3)$ & $-0.027(2)$ & $0.061(8)$ \\
$\mathbf{H}(7)$ & $2 i$ & $0.639(4)$ & $0.320(3)$ & $0.191(3)$ & $0.074(9)$ \\
$\mathbf{H}(3)$ & $2 i$ & $0.875(4)$ & $1.061(3)$ & $0.072(3)$ & $0.063(9)$ \\
$\mathbf{H}(6)$ & $2 i$ & $0.791(4)$ & $0.314(3)$ & $0.036(3)$ & $0.077(9)$ \\
$\mathbf{H}\left(21^{\prime}\right)$ & $2 i$ & $0.943(4)$ & $1.268(4)$ & $0.615(3)$ & $0.068(9)$ \\
$\mathbf{H}\left(22^{\prime}\right)$ & $2 i$ & $0.791(4)$ & $1.133(3)$ & $0.639(3)$ & $0.070(9)$ \\
$\mathbf{H}\left(23^{\prime}\right)$ & $2 i$ & $1.001(5)$ & $1.118(4)$ & $0.621(3)$ & $0.09(1)$ \\
$\mathbf{H}\left(41^{\prime}\right)$ & $2 i$ & $0.209(5)$ & $1.394(4)$ & $0.350(3)$ & $0.09(1)$ \\
$\mathbf{H}\left(42^{\prime}\right)$ & $2 i$ & $0.050(5)$ & $1.257(4)$ & $0.367(3)$ & $0.09(1)$ \\
$\mathbf{H}\left(43^{\prime}\right)$ & $2 i$ & $0.169(5)$ & $1.430(4)$ & $0.479(3)$ & $0.11(1)$ \\
& & & & & \\
\hline
\end{tabular}

\footnotetext{
* Correspondence author (e-mail: shoja @ fordham.edu)
} 
Table 3. Atomic coordinates and displacement parameters (in $\AA^{2}$ ).

\begin{tabular}{|c|c|c|c|c|c|c|c|c|c|c|}
\hline Atom & Site & $x$ & $y$ & $z$ & $U_{11}$ & $U_{22}$ & $U_{33}$ & $U_{12}$ & $U_{13}$ & $U_{23}$ \\
\hline$O(1)$ & $2 i$ & $0.5924(3)$ & $0.7706(2)$ & $0.2380(2)$ & $0.055(1)$ & $0.0394(9)$ & $0.046(1)$ & $0.0207(8)$ & $0.0278(8)$ & $0.0218(8)$ \\
\hline$C(3)$ & $2 i$ & $0.7474(3)$ & $0.9243(3)$ & $0.1317(2)$ & $0.042(1)$ & $0.037(1)$ & $0.040(1)$ & $0.018(1)$ & $0.016(1)$ & $0.020(1)$ \\
\hline$C(4)$ & $2 i$ & $0.8115(3)$ & $0.8040(3)$ & $0.0678(2)$ & $0.038(1)$ & $0.042(1)$ & $0.041(1)$ & $0.018(1)$ & $0.016(1)$ & $0.020(1)$ \\
\hline$O(3)$ & $2 i$ & $0.7973(3)$ & $1.0614(2)$ & $0.1110(2)$ & $0.061(1)$ & $0.045(1)$ & $0.058(1)$ & $0.0292(9)$ & $0.036(1)$ & $0.0315(9)$ \\
\hline$C(10)$ & $2 i$ & $0.7548(3)$ & $0.6627(3)$ & $0.0968(2)$ & $0.039(1)$ & $0.039(1)$ & $0.047(1)$ & $0.017(1)$ & $0.017(1)$ & $0.020(1)$ \\
\hline$C(2)$ & $2 i$ & $0.6417(3)$ & $0.9051(3)$ & $0.2118(2)$ & $0.041(1)$ & $0.037(1)$ & $0.037(1)$ & $0.016(1)$ & $0.014(1)$ & $0.017(1)$ \\
\hline$C(9)$ & $2 i$ & $0.6521(4)$ & $0.6537(3)$ & $0.1835(2)$ & $0.043(1)$ & $0.036(1)$ & $0.043(1)$ & $0.016(1)$ & $0.015(1)$ & $0.017(1)$ \\
\hline$O(4)$ & $2 i$ & $0.9101(3)$ & $0.8232(2)$ & $-0.0048(2)$ & $0.062(1)$ & $0.051(1)$ & $0.062(1)$ & $0.0288(9)$ & $0.040(1)$ & $0.0313(9)$ \\
\hline$C\left(1^{\prime}\right)$ & $2 i$ & $0.5630(3)$ & $1.0173(3)$ & $0.2779(2)$ & $0.042(1)$ & $0.038(1)$ & $0.041(1)$ & $0.017(1)$ & $0.019(1)$ & $0.019(1)$ \\
\hline$C(5)$ & $2 i$ & $0.8053(4)$ & $0.5350(3)$ & $0.0418(3)$ & $0.055(2)$ & $0.048(2)$ & $0.069(2)$ & $0.028(1)$ & $0.033(2)$ & $0.028(1)$ \\
\hline$C\left(4^{\prime}\right)$ & $2 i$ & $0.4038(4)$ & $1.2211(3)$ & $0.4030(2)$ & $0.051(2)$ & $0.041(1)$ & $0.055(2)$ & $0.022(1)$ & $0.027(1)$ & $0.020(1)$ \\
\hline$C\left(2^{\prime}\right)$ & $2 i$ & $0.6401(3)$ & $1.0970(3)$ & $0.4072(2)$ & $0.039(1)$ & $0.039(1)$ & $0.041(1)$ & $0.014(1)$ & $0.015(1)$ & $0.018(1)$ \\
\hline$C(8)$ & $2 i$ & $0.6077(4)$ & $0.5247(3)$ & $0.2197(3)$ & $0.059(2)$ & $0.044(2)$ & $0.059(2)$ & $0.019(1)$ & $0.028(2)$ & $0.028(1)$ \\
\hline$O\left(2^{\prime}\right)$ & $2 i$ & $0.7937(3)$ & $1.0662(2)$ & $0.4618(2)$ & $0.047(1)$ & $0.060(1)$ & $0.043(1)$ & $0.0268(9)$ & $0.0084(8)$ & $0.0165(9)$ \\
\hline$O\left(4^{\prime}\right)$ & $2 i$ & $0.3358(3)$ & $1.3216(2)$ & $0.4743(2)$ & $0.073(1)$ & $0.063(1)$ & $0.068(1)$ & $0.044(1)$ & $0.031(1)$ & $0.019(1)$ \\
\hline$C\left(3^{\prime}\right)$ & $2 i$ & $0.5602(4)$ & $1.1972(3)$ & $0.4689(3)$ & $0.051(2)$ & $0.043(1)$ & $0.039(1)$ & $0.018(1)$ & $0.016(1)$ & $0.011(1)$ \\
\hline$C\left(5^{\circ}\right)$ & $2 i$ & $0.3282(4)$ & $1.1465(3)$ & $0.2752(3)$ & $0.049(2)$ & $0.053(2)$ & $0.054(2)$ & $0.027(1)$ & $0.020(1)$ & $0.027(1)$ \\
\hline$C(6)$ & $2 i$ & $0.7612(5)$ & $0.4077(3)$ & $0.0767(3)$ & $0.066(2)$ & $0.047(2)$ & $0.090(2)$ & $0.031(2)$ & $0.038(2)$ & $0.033(2)$ \\
\hline$C(7)$ & $2 i$ & $0.6659(5)$ & $0.4053(3)$ & $0.1675(3)$ & $0.066(2)$ & $0.044(2)$ & $0.084(2)$ & $0.023(1)$ & $0.032(2)$ & $0.038(2)$ \\
\hline$C\left(6^{\prime}\right)$ & $2 i$ & $0.4093(4)$ & $1.0448(3)$ & $0.2145(2)$ & $0.052(2)$ & $0.049(2)$ & $0.039(1)$ & $0.023(1)$ & $0.017(1)$ & $0.020(1)$ \\
\hline$C\left(22^{\prime}\right)$ & $2 i$ & $0.8895(5)$ & $1.1511(4)$ & $0.5923(3)$ & $0.058(2)$ & $0.063(2)$ & $0.048(2)$ & $0.019(2)$ & $0.004(2)$ & $0.017(2)$ \\
\hline$C\left(44^{\prime}\right)$ & $2 i$ & $0.1777(6)$ & $1.3524(5)$ & $0.4125(4)$ & $0.076(3)$ & $0.069(2)$ & $0.098(3)$ & $0.047(2)$ & $0.044(2)$ & $0.035(2)$ \\
\hline
\end{tabular}

\section{References}

1. Shoja, M.; Sullivan, P.: Crystal structure of 3-hydroxy-2'-methoxyflavone. Z. Kristallogr. NCS 214 (1999) 237-238.

2. Shoja, M.: Crystal structure of 3-hydroxy-6-methoxyflavone. Z. Kristallogr. NCS 213 (1998) 731-732.

3. Shoja, M.; Sullivan, P.; Athanasopoulos, D.; Kabbani, R.: Crystal structure of 3-hydroxy-7-methoxyflavone. Z. Kristallogr. NCS 213 (1998) 579-580.

4. Hall, S. R.; King, G. S. D.; Stewart, J. M. (Eds.): Xtal3.4 User's Manual. University of Western Australia, Lamb, Perth, Australia 1995. 